\title{
Assessing Utilization of the Cerebrospinal Fluid Venereal Disease Research Laboratory Test for Diagnosis of Neurosyphilis: a Cohort Study
}

\author{
Terin T. Sytsma, $M D^{7}$, Elitza S. Theel, $P h D^{2}$, Zelalem Temesgan, $M D^{3}$, and \\ Michel Toledano, $\mathrm{MD}^{4}$
}

'Division of Community Internal Medicine, Mayo Clinic, Rochester, MN, USA; 2Division of Clinical Microbiology, Department of Laboratory Medicine and Pathology, Mayo Clinic, Rochester, MN, USA; ${ }^{3}$ Division of Infectious Disease, Mayo Clinic, Rochester, MN, USA; ${ }^{4}$ Department of Neurology, Mayo Clinic, Rochester, MN, USA.

\begin{abstract}
BACKGROUND: Inappropriate use of diagnostic tests contributes to rising healthcare expenditures, and improving appropriate utilization rates is important for high-value patient care. The Venereal Disease Research Laboratory (VDRL) test performed in cerebrospinal fluid (CSF) has historically been improperly utilized, although there is no recent evaluation of its use in clinical practice. OBJECTIVES: Quantify the rates of appropriate CSFVDRL testing, determine the CSF-VDRL false-positivity rate, and describe the causes of false-positive CSF-VDRL reactivity.
\end{abstract}

DESIGN: Retrospective cohort study

PATIENTS: A total of 32,626 patients with CSF-VDRL testing at one of three Mayo Clinic sites (Rochester, MN; Jacksonville, FL; and Scottsdale, AZ) from January 1, 1994, to February 28, 2018.

MAIN MEASURES: Rate of appropriate CSF-VDRL test utilization from January 1, 2011, to December 31, 2017, and CSF-VDRL true- and false-positivity rates from January 1, 1994, to February 28, 2018.

KEY RESULTS: Among 8553 persons with negative CSFVDRL results, testing was inappropriately ordered for 8399 (98.2\%) of these patients. The word "syphilis" or "neurosyphilis" appeared in the notes of 1184 (13.8\%) individuals with a negative CSF-VDRL result. From January 1994 through February 2018, 33,933 CSF-VDRL tests were performed on 32,626 individual patients. Among the 60 positive CSF-VDRL results, 43 (71.7\%) were true-positives and 17 (28.3\%) were false-positives. All patients with false-positive CSF-VDRL results were tested unnecessarily. Neoplastic meningitis was a common cause of false-positive CSF-VDRL results.

CONCLUSIONS: Inappropriate use of CSF-VDRL testing for the diagnosis of neurosyphilis remains problematic in clinical practice. Following recommended testing algorithms would prevent unnecessary testing and minimize false-positive results.

KEY WORDS: appropriate use; syphilis; non-treponemal; lumbar puncture; neoplastic meningitis.

Prior presentations: A portion of this material was presented at Society for General Internal Medicine, 2019

Received June 1, 2020

Accepted August 10, 2020

Published online August 31, 2020
$\mathrm{J}$ Gen Intern Med 36(1):77-83

DOI: $10.1007 / \mathrm{s} 11606-020-06127-\mathrm{z}$

(c) Society of General Internal Medicine 2020

\section{INTRODUCTION}

Appropriate use of diagnostic testing is crucial to providing high-value healthcare. In 2018, national healthcare expenditures in the USA grew to $\$ 3.6$ trillion, approximately $\$ 11,172$ per person. ${ }^{1}$ The overuse or misuse of screening and diagnostic tests is an important contributor to healthcare expenditures, and with the ever-growing concern that rising healthcare costs are unsustainable, many medical associations emphasize practice patterns that reflect high-value care. ${ }^{2-4}$

The rates of syphilis in the USA have been increasing, but the overall prevalence remains low. In 2018, 35,063 cases $(10.8$ cases per 100,000$)$ of primary or secondary syphilis were reported to the Centers for Disease Control and Prevention (CDC). ${ }^{5}$ Rates of neurosyphilis are much lower, affecting 0.84 to $3.5 \%$ of those diagnosed with primary, secondary, or early latent syphilis. ${ }^{6-8}$ Given the low prevalence of neurosyphilis in the population, it is important to select only individuals with a high pre-test probability of neurosyphilis for CSF-VDRL testing. Dans et al. described the inappropriate use of the Venereal Disease Research Laboratory (VDRL) test performed in cerebrospinal fluid (CSF) for evaluation of neurosyphilis at Johns Hopkins Hospital. In that study, only $28 \%$ of clinicians were considering neurosyphilis as a diagnosis when ordering CSF-VDRL; the vast majority of patients with CSF-VDRL testing had negative or no testing for syphilis performed in blood. ${ }^{9}$ The diagnosis of neurosyphilis is multipronged and frequently relies on the CSF-VDRL test. The VDRL assay is a non-treponemal test which detects antibodies (i.e., reagin) to lipoidal antigens (e.g., cardiolipin, cholesterol, lecithin) released from damaged host cells, and is considered highly specific for neurosyphilis in the right clinical context. ${ }^{10}$, 11

Although there is no gold standard method for the diagnosis of neurosyphilis, multiple published algorithmic approaches and expert reviews recommend performing routine serologic 
Traditional Algorithm

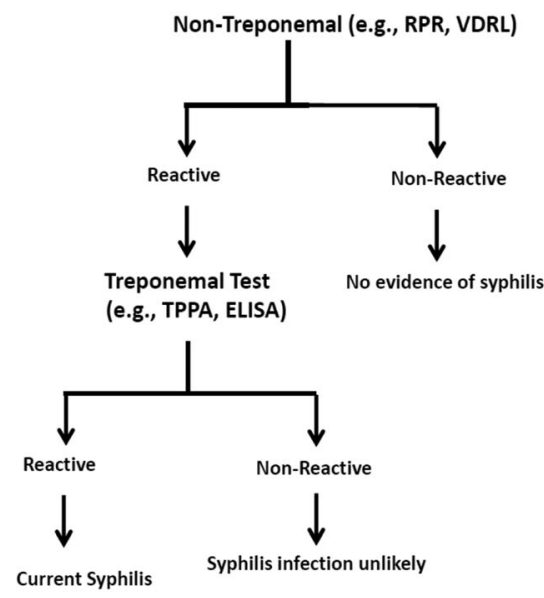

Reverse Algorithm

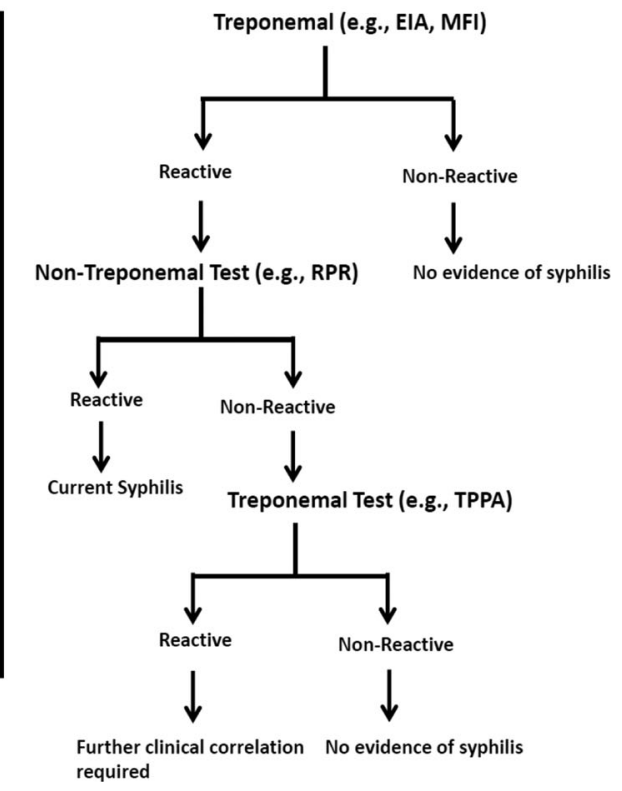

Figure 1 Traditional and reverse algorithms for laboratory detection of syphilis. ${ }^{12,}{ }^{13}$ Abbreviations: EIA, enzyme immunoassay; ELISA, enzyme-linked immunosorbent assay; MFI, multiplex flow immunoassay; RPR, rapid plasma reagin; TPPA, treponema pallidum particle agglutination; VDRL, Venereal Disease Research Laboratory. Note: Non-treponemal testing is not specific and therefore not conclusive for syphilis diagnosis and must be confirmed with a treponemal test in the traditional algorithm. A nonreactive treponemal test indicates that the patient never had syphilis. A reactive treponemal test indicates the patient currently has or has had syphilis, and thus, a reactive treponemal test requires confirmation with a non-treponemal test in the reverse algorithm.

testing for anti-Treponema pallidum antibodies in serum first, using either the traditional or reverse syphilis testing algorithm (Fig. 1). ${ }^{12,13}$ The diagnosis of neurosyphilis requires a positive syphilis serologic test result by either of the aforementioned algorithms; in the absence of such result, CSF-VDRL testing is not recommended given the low probability of infection. ${ }^{14,15}$ Additionally, the CDC recommends testing for neurosyphilis only in at-risk patients with neurologic signs or symptoms consistent with neuroinvasive disease, as well as in those with tertiary syphilis or serologic treatment failure. ${ }^{15}$ The European guidelines also recommend CSF-VDRL testing in HIV-positive patients with CD4+ T cell count $\leq 350 / \mathrm{mm}^{3}$ and/or a serum VDRL or rapid plasma reagen (RPR) titer > $1: 32 .{ }^{14}$ For patients meeting these criteria, although limited in sensitivity, a positive CSF-VDRL test is considered highly specific for neurosyphilis. ${ }^{10,11,14-16}$

False-positive VDRL results in blood have been associated with increased age, pregnancy, malignancy, certain infections (such as HIV), and autoimmune diseases. ${ }^{11,17}$ While blood contamination is known to cause false-positive CSF-VDRL results, other potential causes are not clearly identified. ${ }^{11,17,18}$ There have been multiple case reports of false-positive CSFVDRL results in patients with neoplastic meningitis; however, the frequency of false-positive results secondary to this condition or other causes remains unknown. ${ }^{18-20}$

The objectives of this study were to quantify the rate of appropriate CSF-VDRL testing for neurosyphilis at our institution, identify the CSF-VDRL false-positivity rate, and describe possible cause(s) of false-positive CSF-VDRL reactivity.

\section{METHODS}

Using an institutional electronic medical record database, a retrospective chart review was conducted to identify all instances of CSF-VDRL testing in patients presenting to any one of three Mayo Clinic campuses (Rochester, MN; Jacksonville, FL; and Scottsdale, AZ), between January 1, 2011, and December 31, 2017. Laboratory records during this timeframe were evaluated to identify patients with CSF-VDRL testing performed alone or in combination with a syphilis blood serology. We first evaluated the appropriateness of CSFVDRL testing in those with negative results. CSF-VDRL testing was considered inappropriate in patients without prior syphilis serologic testing, in those with a negative syphilis serologic result in blood (treponemal or non-treponemal), and in those who had CSF-VDRL testing performed prior to syphilis serologic testing in blood. CSF-VDRL testing was deemed appropriate if one or more syphilis tests were positive in serum prior to CSF testing. ${ }^{14,}{ }^{15,21}$ Of note, we did not require that the entire traditional or reverse syphilis screening algorithm $^{12,13}$ (Fig. 1) be interpreted as positive for CSFVDRL testing to be considered appropriate due to the potential for prolonged turnaround time to complete all testing, especially for the reverse algorithm (i.e., 48-72 h), alongside the urgency for clinicians to complete evaluations in select patient care scenarios.

For comparison, we used CPT codes to determine the number of patients who had undergone a diagnostic lumbar puncture during the same time period. Additionally, the medical records of all patients with a negative CSF-VDRL test 
result during this timeframe were reviewed using the search terms "syphilis" and "neurosyphilis" as a way of determining how frequently either of these diagnoses had definitely been considered prior to CSF-VDRL testing being ordered.

Because of the low positivity rate, we expanded our search to January 1, 1994, through February 28, 2018, to identify additional cases of positive CSF-VDRL results. Medical records were reviewed for all patients with a positive CSFVDRL result to determine whether CSF-VDRL testing was appropriate and whether the result was consistent with neurosyphilis. The criteria for a true-positive CSF-VDRL result included the presence of concurrent positive syphilis serologic testing in blood by either the reverse or traditional syphilis testing algorithm (positive treponemal and non-treponemal testing; Fig. 1). A false-positive CSF-VDRL test was defined as a positive result in patients with negative ancillary syphilis testing whose final diagnosis was an infectious or noninfectious process other than neurosyphilis. TTS reviewed all charts of patients with positive CSF-VDRL testing. When there was a question regarding final diagnosis, MT and/or $\mathrm{ZT}$ also reviewed patient charts to come to a final consensus.

Demographic and clinical information for all false-positive CSF-VDRL cases were summarized. Descriptive statistics were used to calculate rates of true-positive and falsepositive CSF-VDRL results.

This study was approved by the Mayo Clinic Institutional Review Board (Approval Number 18-001243).

\section{RESULTS}

From January 1, 2011, to December 31, 2017, 8822 CSFVDRL tests were performed and resulted as negative in 8553 unique patients. Among the 8553 patients with negative CSFVDRL testing, 2855 (33.4\%) had negative syphilis serologic test results, and $155(1.8 \%)$ had at least one positive $(N=147)$ or equivocal $(N=8)$ syphilis serologic blood test result. Three of these 155 patients had serologic testing for syphilis performed in blood after the CSF-VDRL test result was reported. Of the 8553 patients with a negative CSF-VDRL result, 5543 $(64.8 \%)$ did not have any prior syphilis serologic testing performed. Overall, among the 8553 patients with a negative CSF-VDRL result, 8409 (98.3\%) had inappropriate CSFVDRL testing performed according to our pre-defined criteria (Fig. 2). During the same time period, 28,043 individual patients had a diagnostic lumbar puncture, resulting in approximately $30 \%(8,553 / 28,043)$ of patients undergoing a lumbar puncture also having a CSF-VDRL test ordered. The word "syphilis" or "neurosyphilis" appeared in the notes of $13.8 \%$ (1184/8553) of individual patients who had a negative CSFVDRL test result.

From January 1, 1994, to February 28, 2018, 33,933 CSFVDRL tests were performed in 32,626 individual patients. Of these, $33,873(99.8 \%)$ were negative and $60(0.18 \%)$ were positive (Fig. 3). Fifty individual patients accounted for the 60 positive CSF-VDRL results, among which 43 (71.7\%) were classified as true-positives (35 individual patients) with VDRL titers ranging from $1: 1$ to $1: 8$. Seventeen $(28.3 \%)$ were considered falsely positive (15 individual patients) with a similar range in VDRL titers (Fig. 3). The positive predictive value of CSF-VDRL testing in our patient population was $71.7 \%$.

All patients with a true-positive CSF-VDRL result had prior positive syphilis serologic test results in blood, consistent with appropriate CSF-VDRL test utilization, and among these, 16 patients were HIV positive.

The demographics and clinical scenarios of the 15 patients with false-positive CSF-VDRL results are shown in Table 1. None of them had positive treponemal or non-treponemal antibody test results in serum before the CSF-VDRL was obtained. Thus, CSF-VDRL testing was considered inappropriate in all 15 patients. Antibiotics for neurosyphilis were initiated in four $(26.7 \%)$ patients before the diagnosis was reversed, and the positive CSF-VDRL result prompted an infectious disease consult in ten $(66.7 \%)$ cases. Confusion over the diagnosis led to a delay in cancer treatment in at least one patient.

Of the 15 patients with a false-positive CSF-VDRL result, $10(66.7 \%)$ had malignancy affecting the central nervous system. Six $(40.0 \%)$ had meningeal carcinomatosis from metastatic carcinoma (five primary lung cancers and one esophageal cancer), three (20.0\%) were diagnosed with astrocytoma (only two had meningeal involvement), and one (6.7\%) had meningeal lymphomatosis. The remaining five (33.3\%) patients with a false-positive CSF-VDRL had recurrent inflammatory optic neuropathy, multiple sclerosis, Alzheimer's disease, cerebral venous sinus thrombosis, and transient dizziness (Table 1).

\section{DISCUSSION}

CSF-VDRL is considered highly specific for neurosyphilis in the setting of positive serologic testing for syphilis. Currently, there is no data to suggest that a reactive CSF-VDRL in a patient with active syphilis could be falsely positive. Accordingly, current diagnostic guidelines recommend that CSFVDRL only be obtained in patients with positive serological tests for syphilis in blood. ${ }^{8,10}$ Yet $98 \%$ of patients with a negative CSF-VDRL result in our study had either no syphilis testing performed in serum or had a negative serologic result before CSF-VDRL was obtained.

In 1986, Dans and colleagues reported similar levels of inappropriate CSF-VDRL testing. ${ }^{5}$ Their findings suggested that most CSF-VDRL ordering represented automatic "box checking," rather than a thoughtful response to relevant clinical and laboratory data. In our study, the word "syphilis" or "neurosyphilis" appeared in only $13.8 \%$ of charts from patients with negative CSF-VDRL results. This suggests that the diagnosis was rarely considered at the time of test ordering, although admittedly this is hard to establish without more 


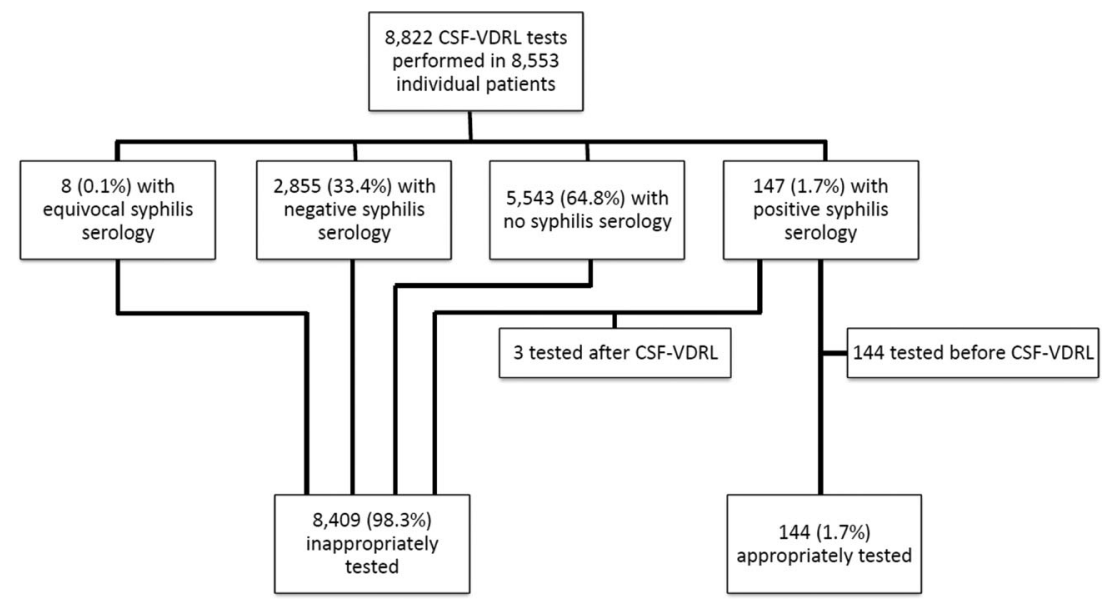

Figure 2 Flow diagram of the appropriateness of CSF-VDRL testing in patients with negative CSF-VDRL results. Abbreviations: CSF, cerebrospinal fluid; VDRL, Venereal Disease Research Laboratory.

careful chart review, and an extensive differential diagnosis may not be documented for every patient in the medical note. Neurosyphilis was not mentioned as a diagnostic consideration in the charts of any of the patients with false-positive CSF-VDRL results before the test was ordered. Despite calls for reform over 30 years ago, ${ }^{9}$ inappropriate CSF-VDRL testing for neurosyphilis remains pervasive in current clinical practice, likely the result of similar "box-checking" or predefined order-sets.

Diagnostic stewardship comprises ordering the right test in the right clinical context to improve patient outcomes. ${ }^{22}$ Strategies to improve diagnostic stewardship in clinical practice include educational campaigns, laboratory protocols, and best practice alerts in the ordering system. ${ }^{23,}{ }^{24}$ All of the patients with true-positive CSF-VDRL results had positive serologic syphilis testing prior to CSF-VDRL collection. This demonstrates the effectiveness of adhering to guidelines to avoid unnecessary testing, thus minimizing the risk of falsepositive results, including overutilization of antibiotics, infectious disease consultation, and delayed cancer treatment.

At our institution, the CSF-VDRL positivity rate over a 24year period was low at $0.18 \%$. Among the positives, $24 \%$ were classified as falsely reactive and all occurred in the setting of inappropriate testing. During the entire study period, not a single positive CSF-VDRL obtained in the absence of positive blood syphilis testing led to a diagnosis of neurosyphilis, highlighting the importance of considering pre-test probability prior to ordering CSF-VDRL, particularly in low-prevalence settings. This false-positivity rate is consistent with a recent study by Zheng et al., which reported a false-positive rate of

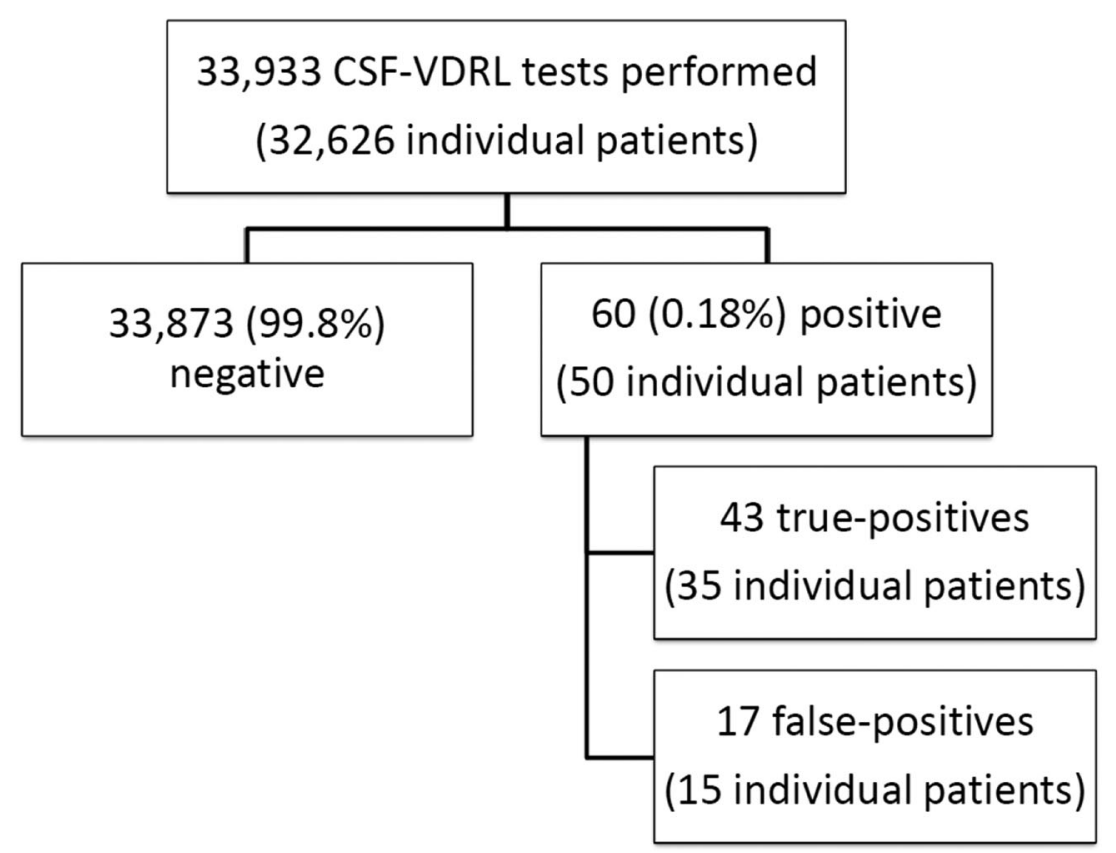

Figure 3 Flow diagram of patients with CSF-VDRL testing from January 1994 through February 2018. Abbreviations: CSF, cerebrospinal fluid; VDRL, Venereal Disease Research Laboratory. 


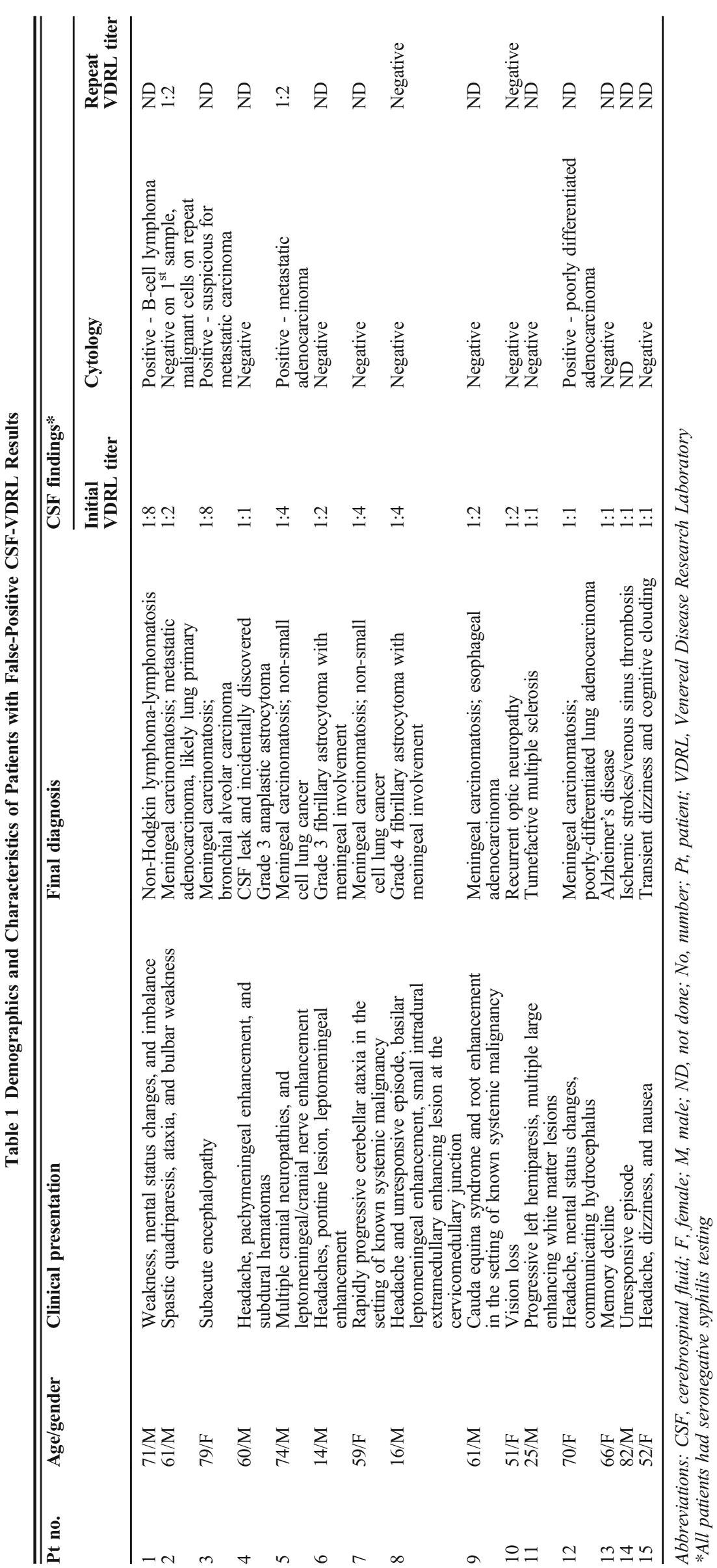


$21 \%$ among CSF-VDRL test results. ${ }^{25}$ However, the cause of the false-positive results differed. In our study, a large percentage of patients with false-positive CSF-VDRL results were ultimately diagnosed with neoplastic meningitis, mostly meningeal carcinomatosis associated with primary lung cancer. Zheng et al. did not find any cases of neoplastic meningitis. ${ }^{25}$ The difference between our findings is likely due in part to different populations and/or practice patterns. The Zheng study was conducted in Singapore, and importantly, their study was conducted over a 3-year period, whereas we analyzed data spanning 24 years. It is possible that a similar pattern may have emerged in the Zheng study had they analyzed data over a longer period of time.

Although referral bias may have accounted for the overrepresentation of neoplastic meningitis in our cohort, inappropriate CSF-VDRL testing appears to be a pervasive practice across the institution, and it is unlikely that the test is disproportionately ordered in patients with underlying malignancy. In addition, previous case reports and case series have reported false-positive CSF-VDRL results in patients with meningeal carcinomatosis from lung cancer. ${ }^{18-20}$ Non-treponemal assays detect antibodies to a combination of antigens primarily released from damaged host cells, including cardiolipin, lecithin, and cholesterol. ${ }^{17}$ The presence of these antigens in other disease states (e.g., systemic lupus erythematosus, HIV) is known to cause biologic false-positive non-treponemal results in serum; however, less is known about biologic false-positive results from non-treponemal tests in CSF. ${ }^{17,26}$

The current study has several limitations. First, our results are from a tertiary care center; some of the patients without syphilis serologic testing in blood may have had testing done at an outside institution prior to referral. However, given the large number of negative serologic syphilis tests in patients with negative CSF-VDRL testing, the pattern of inappropriate CSF-VDRL test use would likely stand even if some patients had serologic testing available at other institutions. Second, practice patterns at tertiary centers may differ from that of smaller community centers. Given that the evaluation for and treatment of neurosyphilis often requires consultation with infectious disease, the majority of patients for whom neurosyphilis is considered are likely cared for at larger care centers. Further research could examine interventions aimed at decreasing the inappropriate use of CSF-VDRL testing, such as laboratory protocols requiring positive blood syphilis serologic test results prior to testing CSF-VDRL.

In conclusion, our findings indicate that more needs to be done to ensure that diagnostic algorithms are followed to prevent unnecessary testing and minimize the risk of false-positive results. While test ordering practices may be controlled to an extent by the performing laboratory, ordering clinicians are encouraged to adhere to published guidelines and expert recommendations with respect to appropriate test utilization, including by routinely vetting pre-established test order sets.
Corresponding Author: Michel Toledano, MD; Department of Neurology, Mayo Clinic, Rochester, MN, USA (e-mail: toledano. michel@mayo.edu).

\section{Compliance with Ethical Standards:}

This research was reviewed and approved by the Mayo Clinic Institutional Review Board (Approval Number 18-001243).

Conflict of Interest: The authors declare that they do not have a conflict of interest.

\section{REFERENCES}

1. United States Centers for Medicare \& Medicaid Services. NHE Fact Sheet 2019 [updated April 26, 2019]. Available from: https://www.cms.gov/ Research-Statistics-Data-and-Systems/Statistics-Trends-and-Reports / NationalHealthExpendData/NHE-Fact-Sheet.html. Accessed 14 Nov 2019.

2. Cassel CK, Guest JA. Choosing Wisely: Helping Physicians and Patients Make Smart Decisions About Their Care. JAMA. 2012;307(17):1801-2. https://doi.org/10.1001/jama.2012.476.

3. Gaseem A, Alguire P, Dallas P, Feinberg LE, Fitzgerald FT, Horwitch C, et al. Appropriate use of screening and diagnostic tests to foster highvalue, cost-conscious care. Ann Intern Med. 2012;156(2):147-9.

4. Rao VM, Levin DC. The overuse of diagnostic imaging and the Choosing Wisely initiative. Ann Intern Med. 2012;157(8):574-6.

5. Centers for Disease Control and Prevention. Sexually Transmitted Disease Surveillance 2018 Atlanta: US Department of Health and Human Services Available from: https://www.cdc.gov/std/stats/. Accessed 19 Dec 2019.

6. de Voux A, Kidd S, Torrone EA. Reported Cases of Neurosyphilis Among Early Syphilis Cases-United States, 2009 to 2015. Sex Transm Dis. 2018;45(1):39-41. https://doi.org/10.1097/OLQ.0000000000000687.

7. Dombrowski JC, Pedersen R, Marra CM, Kerani RP, Golden MR. Prevalence estimates of complicated syphilis. Sex Transm Dis. 2015;42(12):702-4.

8. Landry T, Smyczek P, Cooper R, Gratrix J, Bertholet L, Read R, et al. Retrospective review of tertiary and neurosyphilis cases in Alberta, 19732017. BMJ Open. 2019;9(6):e025995.

9. Dans PE, Cafferty L, Otter SE, Johnson RJ. Inappropriate use of the cerebrospinal fluid Venereal Disease Research Laboratory (VDRL) test to exclude neurosyphilis. Ann Intern Med. 1986;104(1):86-9.

10. Ho EL, Marra CM. Treponemal Tests for Neurosyphilis-Less Accurate Than What We Thought? Sex Transm Dis. 2012;39(4):298.

11. Singh AE, Romanowski B. Syphilis: review with emphasis on clinical, epidemiologic, and some biologic features. Clin Microbiol Rev. 1999; 12(2):187-209.

12. Binnicker MJ. Which algorithm should be used to screen for syphilis? Curr Opin Infect Dis 2012;25(1):79-85.

13. Loeffelholz MJ, Binnicker MJ. Point-Counterpoint: It is time to use treponema-specific antibody screening tests for diagnosis of syphilis. $\mathrm{J}$ of Clin Microbiol. 2012;50(1):2-6

14. Janier M, Hegyi V, Dupin N, Unemo M, Tiplica GS, Potocnik M, et al. 2014 European guideline on the management of syphilis. J Eur Acad Dermatol Venereol. 2014;28(12):1581-93. https://doi.org/10.1111/jdv. 12734 .

15. Workowski KA, Bolan GA. Sexually transmitted diseases treatment guidelines, 2015. MMWR Recomm Rep.. 2015;64(Rr-03):1-137.

16. Davis LE, Schmitt JW. Clinical significance of cerebrospinal fluid tests for neurosyphilis. Ann Neurol. 1989;25(1):50-5.

17. Kennedy EJ, Creighton ET. VENEREAL DISEASE RESEARCH LABORATORY (VDRL) SLIDE TEST. Manual of Tests for Syphilis 9th Edition 1998.

18. Gran A, Cunha BA. False-Positive Venereal Disease Research Laboratory Tests in the Cerebrospinal Fluid With Meningeal Carcinomatosis. Infect Dis Clin Pract. 2015;23(3):e13-e4.

19. Madiedo G, Ho K-C, Walsh P. False-positive VDRL and FTA in cerebrospinal fluid. JAMA. 1980;244(7):688-9.

20. Tung Y-J, Hsieh M-h, Lin C-Y, Chen Y-H, Huang C-H. False positive venereal disease research laboratory of cerebrospinal fluid. J Microbiol Immunol Infect. 2015;48(2):S130. 
21. Braxton J, Davis DW, Emerson B, Flagg EW, Grey J, Grier L, et al. Sexually transmitted disease surveillance 2017. 2018. https://doi.org/ 10.15620/cdc.59237.

22. Gomez-Puerta JA, Cervera R, Espinosa G, Aguilo S, Bucciarelli S, Ramos-Casals M, et al. Antiphospholipid antibodies associated with malignancies: clinical and pathological characteristics of 120 patients. Semin Arthritis Rheum. 2006;35(5):322-32. https://doi.org/10.1016/j. semarthrit.2005.07.003.

23. Jun, T, Kwang, H, Mou, E, Berube, C, Bentley, J, Shieh, L, \& Hom, J. An electronic best practice alert based on choosing wisely guidelines reduces thrombophilia testing in the outpatient setting. $J$ Gen Intern Med. 2019;34(1): 29.

24. Morgan, DJ, Malani, P, \& Diekema, DJ. Diagnostic stewardship-leveraging the laboratory to improve antimicrobial use. JAMA. 2017;318(7): 607-608.
25. Zheng S, Lin R, Chan $\mathbf{Y}$, Ngan C. Biological false-positive venereal disease research laboratory test in cerebrospinal fluid in the diagnosis of neurosyphilis-a case-control study. J Eur Acad Dermatol Venereol. 2018;32(3):474-81.

26. Geusau A, Kittler H, Hein U, Dangl-Erlach E, Sting1 G, Tschachler E. Biological false-positive tests comprise a high proportion of Venereal Disease Research Laboratory reactions in an analysis of 300,000 sera. Int J STD AIDS. 2005; 16(11):722-6.

Publisher's Note Springer Nature remains neutral with regard to jurisdictional claims in published maps and institutional affiliations. 